\title{
Observer-Based Robust Passive Control for a Class of Uncertain Neutral Systems: An Integral Sliding Mode Approach
}

\author{
Ruiping Xu, ${ }^{1,2}$ Zhen Liu, ${ }^{3}$ Cunchen Gao, ${ }^{3}$ and Huimin Xiao ${ }^{4}$ \\ ${ }^{1}$ College of Information Science and Engineering, Ocean University of China, Qingdao 266100, China \\ ${ }^{2}$ College of Mathematics, Qingdao University, Qingdao 266071, China \\ ${ }^{3}$ School of Mathematical Sciences \& College of Physical and Environmental Oceanography, Ocean University of China, \\ Qingdao 266100, China \\ ${ }^{4}$ Institute of Information and Systems Engineering, Henan University of Economics and Law, Zhengzhou 450002, China \\ Correspondence should be addressed to Zhen Liu; liuzhen8788@163.com
}

Received 20 December 2014; Revised 29 April 2015; Accepted 30 April 2015

Academic Editor: Onur Toker

Copyright (C) 2015 Ruiping Xu et al. This is an open access article distributed under the Creative Commons Attribution License, which permits unrestricted use, distribution, and reproduction in any medium, provided the original work is properly cited.

The problem of integral sliding mode control (ISMC) with passivity is investigated for a class of uncertain neutral systems with time-varying delay (NTSTD) and external disturbance. The system states are unavailable. An ISMC strategy is proposed based on the state estimate. By employing a novel sliding functional, a new sufficient criterion of robust asymptotic stability and passivity for both the error system and the sliding mode (SM) dynamic system is derived via linear matrix inequality (LMI) technique. Then, a SM controller is synthesized to guarantee the reachability of the sliding surface predefined in the state estimate space. Finally, a numerical example shows the feasibility and superiority of the obtained result.

\section{Introduction}

In the mathematical modeling of a real control system, state feedback control is a powerful tool when the full information of the system states is assumed to be accessible. However, in practice, this is often not possible, and state feedback control may fail to guarantee the stability when some of the system states are not measurable. To circumvent this drawback, one way is to design a direct output feedback control. Another way, that is the observer-based controller, is also utilized to improve the system performance and stabilize the unstable systems. Therefore, the observer-based control for complex systems has become an active research topic in control theory [1-6].

SMC has been widely recognized as an effective robust control strategy for incompletely modelled and uncertain systems. The SMC system has various attractive features such as fast response, good transient performance, and robustness in face of uncertainties and external disturbances. Recently, sliding mode observers have been used to estimate the states of discrete or continuous systems. They have a special function of dealing with nonlinearity and uncertainties.
Because there will be some term injected into the observer depending on the output error estimation, which enables the observer to reject the effect of external disturbance and make the error dynamic system stable. Over the past decades, a rich literature has been dedicated to the design of the sliding mode observers [7-14].

On the other hand, since time delays are frequently encountered in practical engineering and often result in poor performance or even instability of system, analysis of robust stability and stabilization of uncertain time delay system has attracted many researchers' attention. In the more general case of NTSTD, where the time delay may be available in both position and velocity of system, the stability and stabilization criterion based upon LMI technique have been obtained for both the delay-independent [15] and the delay-dependent cases in the literature ([16-23] and the references therein). As shown in [5], the presence of a delayed argument in the state derivatives makes the design difficult, which results from the neutral root chain (infinite spectrum). Generally, the authors also assume that there is no unstable neutral root chain or use a derivative feedback to assign the unstable roots to the lefthand side of the complex plane. 
Note that unlike simple retarded systems neutral systems may be particularly sensitive to time delays and can be easily destabilized. Specifically, the SMC problem for NTSTD with uncertainties in both the state matrices and the input matrix is considered in [24-27], which gives sufficient conditions in terms of LMIs guaranteeing the closed-loop system to be asymptotically stable, respectively. However, in these papers, the key requirement is that the states of the system are available. Moreover, the observer-based SMC problem for a class of uncertain nonlinear NTSTD is investigated in [28]. A sliding mode observer is designed, based on which an observer-based controller is synthesized by using the SMC theory combined with the reaching law technique; the reachability of the sliding surfaces defined in both the state estimate space and the state estimation error space is solved, respectively. To the best of our knowledge, the sliding mode observer design problem and the observerbased control problem for uncertain NTSTD have not yet been fully investigated in the literature and remain to be important and challenging.

Motivated by the aforementioned factors, a new approach of SMC combined with passive control is proposed for stabilizing the uncertain NTSTD subjected to external disturbance and unmeasurable states in this paper. A state observer is designed to generate the estimate of system states, based on which a SMC scheme is developed to stabilize the closedloop system. A sufficient condition of the passivity and robust asymptotic stability is derived for the overall closed-loop system composed of the observer SM dynamic system and the state estimate error dynamic system via LMI. The proposed SMC can guarantee the reachability of the sliding surface in state estimate space in finite time. Finally, an example is presented to show the effectiveness of the proposed method.

\section{Problem Formulation}

The form of the uncertain NTSTD with external disturbance studied in this paper is

$$
\begin{aligned}
\dot{\mathbf{x}}(t)-\mathbf{D} \dot{\mathbf{x}}(t-\tau)= & {[\mathbf{A}+\Delta \mathbf{A}(t)] \mathbf{x}(t) } \\
& +\left[\mathbf{A}_{d}+\Delta \mathbf{A}_{d}(t)\right] \mathbf{x}(t-d(t)) \\
& +\mathbf{B u}(t)+\mathbf{G}_{w} \mathbf{w}(t), \\
\mathbf{z}(t)= & \mathbf{C} \mathbf{x}(t), \\
\mathbf{x}(s)= & \boldsymbol{\varphi}(s), \\
\dot{\mathbf{x}}(s)= & \psi(s) ; \\
& s \in[-\bar{d}, 0],
\end{aligned}
$$

where $\mathbf{x}(t) \in \mathfrak{R}^{n}$ is the system state, $\mathbf{u}(t) \in \mathfrak{R}^{m}$ is the control input, $\mathbf{z}(t) \in \mathfrak{R}^{q}$ is the controlled output, and $\boldsymbol{\varphi}(t)$ and $\psi(t)$ are vector valued functions representing the initial conditions. $\mathbf{w}(t)$ is the exogenous input which belongs to $\mathbb{L}_{2}[0, T]$. The scalar $\tau$ is a real constant neutral-term time delay and $d(t)$ is the time-varying delay satisfying $0 \leq$ $d(t) \leq d, \dot{d}(t) \leq \mu<1$ for known constants $d, \mu$, and $\bar{d}=\max \{\tau, d\} . \mathbf{A}, \mathbf{A}_{d}, \mathbf{B}, \mathbf{C}$, and $\mathbf{D}$ are known system matrices with appropriate dimensions, and $\mathbf{B}$ has full column rank.

The following assumptions and lemmas are necessary for future discussion.

Assumption 1. (A, B) is completely controllable.

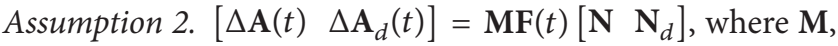
$\mathbf{N}, \mathbf{N}_{d}$ are known matrices of appropriate dimensions and $\mathbf{F}(t)$ is an unknown matrix function which is continuous with respect to time $t$ and satisfies $\mathbf{F}^{\mathrm{T}}(t) \mathbf{F}(t) \leq \mathbf{I}$.

Lemma 3 (see [9]). Let $\mathbf{D}, \mathbf{E}$, and $\mathbf{F}(t)$ be real matrices of appropriate dimensions with $\mathbf{F}(t)$ satisfying $\mathbf{F}^{\mathrm{T}}(t) \mathbf{F}(t) \leq \mathbf{I}$ and a scalar $\varepsilon>0$; the following inequality

$$
\mathbf{D F}(t) \mathbf{E}+\mathbf{E}^{\mathrm{T}} \mathbf{F}^{\mathrm{T}}(t) \mathbf{D}^{\mathrm{T}} \leq \varepsilon \mathbf{D D}^{\mathrm{T}}+\boldsymbol{\varepsilon}^{-1} \mathbf{E}^{\mathrm{T}} \mathbf{E}
$$

is always satisfied.

\section{Observer-Based Sliding Mode Control}

In this section, an observer is introduced to generate the estimate of the unmeasured state. And then a SM controller will be synthesized based on the state estimate. Furthermore, a sufficient condition of the passivity and robust asymptotic stability is derived for the overall closed-loop system composed of the observer SM dynamic system and the error dynamic system via LMI.

3.1. Robust Observer Design. The following state observer is utilized to estimate the state of the uncertain NTSTD (1):

$$
\begin{aligned}
\dot{\widehat{\mathbf{x}}}(t)-\mathbf{D} \dot{\widehat{\mathbf{x}}}(t-\tau)= & \mathbf{A} \widehat{\mathbf{x}}(t)+\mathbf{A}_{d} \widehat{\mathbf{x}}(t-d(t))+\mathbf{B u}(t) \\
& +\mathbf{L}(\mathbf{z}(t)-\mathbf{C} \widehat{\mathbf{x}}(t)), \\
\widehat{\mathbf{z}}(t)= & \mathbf{C} \widehat{\mathbf{x}}(t),
\end{aligned}
$$

where $\mathbf{L} \in \mathfrak{R}^{n \times q}$ is the observer gain to be designed later.

Define the state error $\mathbf{e}(t)=\mathbf{x}(t)-\widehat{\mathbf{x}}(t)$; then the corresponding error system can be described by

$$
\begin{aligned}
\dot{\mathbf{e}}(t)-\mathbf{D} \dot{\mathbf{e}}(t-\tau)= & {[\mathbf{A}-\mathbf{L} \mathbf{C}+\Delta \mathbf{A}(t)] \mathbf{e}(t) } \\
& +\left[\mathbf{A}_{d}+\Delta \mathbf{A}_{d}(t)\right] \mathbf{e}(t-d(t)) \\
& +\mathbf{G}_{w} \mathbf{w}(t)+\Delta \mathbf{A}(t) \widehat{\mathbf{x}}(t) \\
& +\Delta \mathbf{A}_{d}(t) \widehat{\mathbf{x}}(t-d(t)), \\
\mathbf{z}_{e}(t)= & \mathbf{C e}(t),
\end{aligned}
$$

where $\mathbf{z}_{e}(t)$ is the output of the error system.

Remark 4. It can be seen from (4) that the error dynamic system corresponds to an uncertain NTSTD and is dependent on the state estimate. This means that the robust stability analysis of the error dynamic system is not independent of the observer system. 
In the state estimate space, a sliding functional is chosen as follows:

$$
\mathbf{s}(t, \widehat{\mathbf{x}})=\boldsymbol{\sigma}(t)+\mathbf{G}[\widehat{\mathbf{x}}(t)-\mathbf{D} \widehat{\mathbf{x}}(t-\tau)],
$$

with $\dot{\boldsymbol{\sigma}}(t)=-\mathbf{G}(\mathbf{A}+\mathbf{B K}) \widehat{\mathbf{x}}(t)-\mathbf{G A}_{d} \widehat{\mathbf{x}}(t-d(t))$, where the matrix $\mathbf{K}$ is to be determined later. Particularly, the matrix $\mathbf{G}$ is to be chosen such that GB is nonsingular.

According to the sliding mode control theory, it follows from $\dot{\mathbf{s}}(t, \widehat{\mathbf{x}})=\mathbf{0}$ that the equivalent control law can be obtained as

$$
\mathbf{u}_{\mathrm{eq}}=\mathbf{K} \widehat{\mathbf{x}}(t)-(\mathbf{G B})^{-1} \mathbf{G L}[\mathbf{z}(t)-\mathbf{C} \widehat{\mathbf{x}}(t)] .
$$

Substituting (6) into the observer system (3) yields the SM dynamic system in the state estimate space:

$$
\begin{aligned}
\dot{\widehat{\mathbf{x}}}(t)-\mathbf{D} \dot{\widehat{\mathbf{x}}}(t-\tau)= & (\mathbf{A}+\mathbf{B K}) \widehat{\mathbf{x}}(t)+\mathbf{A}_{d} \widehat{\mathbf{x}}(t-d(t)) \\
& +\overline{\mathbf{B}} \mathbf{L C e}(t)
\end{aligned}
$$

where $\overline{\mathbf{B}}=\mathbf{I}-\mathbf{B}(\mathbf{G B})^{-1} \mathbf{G}$.

Thus, the stability of the overall closed-loop systems with (3) and (4) will be analyzed through the error system (4) and SM dynamic system (7).

3.2. Analysis of Robust Stability. Here, we introduce the passive performance measure as follows.

Definition 5. The overall closed-loop system composed of (4) and (7) is said to be robust asymptotically stable and passive, if the following two requirements are satisfied:

(I) system (4) and (7) with $\mathbf{w}(t)=\mathbf{0}$ is robust asymptotically stable;

(II) under zero initial conditions, there exists a scalar $\gamma>$ 0 such that

$$
2 \int_{0}^{t^{*}} \mathbf{w}^{\mathrm{T}}(t) \mathbf{z}_{e}(t) \mathrm{d} t \geq-\gamma \int_{0}^{t^{*}} \mathbf{w}^{\mathrm{T}}(t) \mathbf{w}(t) \mathrm{d} t
$$

for all $t^{*}>0$.

Next, the following sufficient condition for the passivity and robust asymptotic stability for the overall closed-loop system composed of the observer SM dynamic system (7) and the error dynamic system (4) is derived via LMI.

Theorem 6. Given a scalar $\gamma>0$. Unmeasured state of the uncertain NTSTD (1) can be estimated by the observer (3). The sliding functional in state estimate space is chosen as (5). If there exist symmetric positive definite matrices $\mathbf{P}>\mathbf{0}, \mathbf{Q}>\mathbf{0}, \mathbf{R}>\mathbf{0}$,
$\mathbf{S}>\mathbf{0}$, matrices $\mathbf{L}, \mathbf{K}$, and scalars $\varepsilon_{i}>0(i=1, \ldots, 4)$ such that the following linear matrix inequality holds

$$
\left[\begin{array}{cccccccc}
\boldsymbol{\Xi}_{11} & \boldsymbol{\Xi}_{12} & \mathbf{A}_{d} & \boldsymbol{\Xi}_{14} & \mathbf{0} & \mathbf{0} & \boldsymbol{\Xi}_{17} & \mathbf{J}_{2} \\
* & \boldsymbol{\Xi}_{22} & \mathbf{0} & \boldsymbol{\Xi}_{24} & \mathbf{0} & \mathbf{0} & \mathbf{\Xi}_{27} & \mathbf{0} \\
* & * & \boldsymbol{\Xi}_{33} & \mathbf{0} & \mathbf{0} & \mathbf{0} & \mathbf{0} & \mathbf{0} \\
* & * & * & \boldsymbol{\Xi}_{44} & \boldsymbol{\Xi}_{45} & \mathbf{A}_{d} & \mathbf{0} & \mathbf{0} \\
* & * & * & * & \boldsymbol{\Xi}_{55} & \mathbf{0} & \mathbf{0} & \mathbf{0} \\
* & * & * & * & * & \boldsymbol{\Xi}_{66} & \mathbf{0} & \mathbf{0} \\
* & * & * & * & * & * & -\gamma \mathbf{I} & \mathbf{0} \\
* & * & * & * & * & * & * & -\mathbf{J}_{1}
\end{array}\right]<\mathbf{0}
$$

where

$$
\begin{aligned}
& \boldsymbol{\Xi}_{11}=\operatorname{sym}\{\mathbf{A}-\mathbf{L C}\}+\mathbf{P}+\mathbf{Q}+2 \beta \mathbf{I}+\varepsilon_{1} \mathbf{N}^{\mathrm{T}} \mathbf{N}, \\
& \Xi_{12}=\Theta_{12} \text {, } \\
& \boldsymbol{\Xi}_{22}=\boldsymbol{\Theta}_{22} \text {, } \\
& \Xi_{33}=\boldsymbol{\Theta}_{33}, \\
& \Xi_{45}=\Theta_{45} \text {, } \\
& \boldsymbol{\Xi}_{44}=\operatorname{sym}\{\mathbf{A}+\mathbf{B K}\}+\mathbf{R}+\mathbf{S}+2 \beta \mathbf{I}+\varepsilon_{3} \mathbf{N}^{\mathrm{T}} \mathbf{N}, \\
& \Xi_{55}=\boldsymbol{\Theta}_{55} \text {, } \\
& \Xi_{66}=\boldsymbol{\Theta}_{66}, \\
& \mathbf{\Xi}_{14}=(\overline{\mathbf{B}} \mathbf{L C})^{\mathrm{T}} \text {, } \\
& \mathbf{J}_{1}=\operatorname{diag}\left\{\varepsilon_{1} \mathbf{I}, \varepsilon_{2} \mathbf{I}, \varepsilon_{3} \mathbf{I}, \varepsilon_{4} \mathbf{I}\right\}, \\
& \mathbf{\Xi}_{24}=(\overline{\mathbf{B}} \mathbf{L} \mathbf{C D})^{\mathrm{T}} \text {, } \\
& \mathbf{J}_{2}=\left[\begin{array}{llll}
\mathbf{M} & \mathbf{M} & \mathbf{M} & \mathbf{M}
\end{array}\right], \\
& \mathbf{\Xi}_{17}=-\mathbf{C}^{\mathrm{T}}+\mathbf{G}_{w} \text {, } \\
& \boldsymbol{\Xi}_{27}=-(\mathbf{C D})^{\mathrm{T}} \text {, }
\end{aligned}
$$

then, the overall closed-loop system with (4) and (7) is said to be robust asymptotically stable and passive.

Proof. Define

$$
\begin{aligned}
& \mathscr{D}\left(e_{t}\right)=\mathbf{e}(t)-\mathbf{D e}(t-\tau), \\
& \mathscr{D}\left(\widehat{\mathbf{x}}_{t}\right)=\widehat{\mathbf{x}}(t)-\mathbf{D} \widehat{\mathbf{x}}(t-\tau) .
\end{aligned}
$$

Choose the Lyapunov functional candidate

$$
\begin{aligned}
\mathbf{V}(t)= & \mathbf{V}_{1}(t)+\mathbf{V}_{2}(t)+\mathbf{V}_{3}(t)+\mathbf{V}_{4}(t)+\mathbf{V}_{5}(t) \\
& +\mathbf{V}_{6}(t),
\end{aligned}
$$


where

$$
\begin{aligned}
& \mathbf{V}_{1}(t)=\mathscr{D}^{\mathrm{T}}\left(e_{t}\right) \mathscr{D}\left(e_{t}\right), \\
& \mathbf{V}_{2}(t)=\int_{t-d(t)}^{t} e^{2 \beta(s-t)} \mathbf{e}^{\mathrm{T}}(s) \mathbf{P e}(s) \mathrm{d} s, \\
& \mathbf{V}_{3}(t)=\int_{t-\tau}^{t} e^{2 \beta(s-t)} \mathbf{e}^{\mathrm{T}}(s) \mathbf{Q e}(s) \mathrm{d} s, \\
& \mathbf{V}_{4}(t)=\mathscr{D}^{\mathrm{T}}\left(\widehat{x}_{t}\right) \mathscr{D}\left(\widehat{x}_{t}\right), \\
& \mathbf{V}_{5}(t)=\int_{t-d(t)}^{t} e^{2 \beta(s-t)} \widehat{\mathbf{x}}^{\mathrm{T}}(s) \mathbf{R} \widehat{\mathbf{x}}(s) \mathrm{d} s, \\
& \mathbf{V}_{6}(t)=\int_{t-\tau}^{t} e^{2 \beta(s-t)} \widehat{\mathbf{x}}^{\mathrm{T}}(s) \mathbf{S} \widehat{\mathbf{x}}(s) \mathrm{d} s .
\end{aligned}
$$

Firstly, we consider the overall closed-loop system with $\mathbf{w}(t)=\mathbf{0}$. It can be derived from (4) and (7) that

$$
\begin{aligned}
& \dot{\mathbf{V}}_{1}(t)=2 \mathscr{D}^{\mathrm{T}}\left(e_{t}\right)\{[\mathbf{A}-\mathbf{L C}+\Delta \mathbf{A}(t)] \mathbf{e}(t) \\
&+ {\left[\mathbf{A}_{d}+\Delta \mathbf{A}_{d}(t)\right] \mathbf{e}(t-d(t))+\Delta \mathbf{A}(t) \widehat{\mathbf{x}}(t) } \\
&+\left.\Delta \mathbf{A}_{d}(t) \widehat{\mathbf{x}}(t-d(t))\right\} \\
& \dot{\mathbf{V}}_{2}(t)=\mathbf{e}^{\mathrm{T}}(t) \mathbf{P e}(t)-e^{-2 \beta d(t)}(1-\dot{d}(t)) \cdot \mathbf{e}^{\mathrm{T}}(t \\
&-d(t)) \mathbf{P e}(t-d(t))-2 \beta \mathbf{V}_{2}(t) \leq \mathbf{e}^{\mathrm{T}}(t) \mathbf{P e}(t) \\
&-e^{-2 \beta d}(1-\mu) \cdot \mathbf{e}^{\mathrm{T}}(t-d(t)) \mathbf{P e}(t-d(t)) \\
&-2 \beta \mathbf{V}_{2}(t), \\
& \dot{\mathbf{V}}_{3}(t)=\mathbf{e}^{\mathrm{T}}(t) \mathbf{Q} \mathbf{e}(t)-e^{-2 \beta \tau} \cdot \mathbf{e}^{\mathrm{T}}(t-\tau) \mathbf{Q} \mathbf{e}(t-\tau) \\
&-2 \beta \mathbf{V}_{3}(t), \\
& \dot{\mathbf{V}}_{4}(t)=2 \mathscr{D}^{\mathrm{T}}\left(\widehat{x}_{t}\right)\left\{(\mathbf{A}+\mathbf{B K}) \widehat{\mathbf{x}}(t)+\mathbf{A}_{d} \widehat{\mathbf{x}}(t-d(t))\right. \\
&+\overline{\mathbf{B}}_{\mathbf{L} C \mathbf{e}(t)\}} \\
& \dot{\mathbf{V}}_{5}(t)=\widehat{\mathbf{x}}^{\mathrm{T}}(t) \mathbf{R} \widehat{\mathbf{x}}(t)-(1-\dot{d}(t)) e^{-2 \beta d(t)} \cdot \widehat{\mathbf{x}}^{\mathrm{T}}(t \\
&-d(t)) \mathbf{R} \widehat{\mathbf{x}}(t-d(t))-2 \beta \mathbf{V}_{5}(t) \leq \widehat{\mathbf{x}}^{\mathrm{T}}(t) \mathbf{R} \widehat{\mathbf{x}}(t) \\
&-e^{-2 \beta d}(1-\mu) \cdot \widehat{\mathbf{x}}^{\mathrm{T}}(t-d(t)) \mathbf{R} \widehat{\mathbf{x}}(t-d(t)) \\
&-2 \beta \mathbf{V}_{5}(t) \\
& \dot{\mathbf{V}}_{6}(t)=\widehat{\mathbf{x}}^{\mathrm{T}}(t) \mathbf{S} \widehat{\mathbf{x}}(t)-e^{-2 \beta \tau} \cdot \widehat{\mathbf{x}}^{\mathrm{T}}(t-\tau) \mathbf{S} \widehat{\mathbf{x}}(t-\tau) \\
&-2 \beta \mathbf{V}_{6}(t)
\end{aligned}
$$

So, we have

$$
\begin{aligned}
\dot{\mathbf{V}}(t) \leq & \mathscr{D}^{\mathrm{T}}\left(\mathbf{e}_{t}\right)[\operatorname{sym}\{(\mathbf{A}-\mathbf{L C})\}+\mathbf{P}+\mathbf{Q}] \mathscr{D}\left(\mathbf{e}_{t}\right) \\
& +2 \mathscr{D}^{\mathrm{T}}\left(e_{t}\right)(\mathbf{A}-\mathbf{L C}) \mathbf{D e}(t-\tau)+2 \mathscr{D}^{\mathrm{T}}\left(\mathbf{e}_{t}\right) \\
& \cdot \mathbf{A}_{d} \mathbf{e}(t-d(t))+2 \mathscr{D}^{\mathrm{T}}\left(\mathbf{e}_{t}\right)(\mathbf{P}+\mathbf{Q}) \mathbf{D} \\
& \cdot \mathbf{e}(t-\tau)+\mathbf{e}^{\mathrm{T}}(t-\tau) \mathbf{D}^{\mathrm{T}}(\mathbf{P}+\mathbf{Q}) \mathbf{D e}(t-\tau)
\end{aligned}
$$

$$
\begin{aligned}
& -e^{-2 \beta \tau} \mathbf{e}^{\mathrm{T}}(t-\tau) \mathbf{Q} \mathbf{e}(t-\tau)-(1-\mu) e^{-2 \beta d} \\
& \cdot \mathbf{e}^{\mathrm{T}}(t-d(t)) \mathbf{P e}(t-d(t))+\mathscr{D}^{\mathrm{T}}\left(\widehat{\mathbf{x}}_{t}\right) \\
& \cdot[\operatorname{sym}\{(\mathbf{A}+\mathbf{B K})\}+\mathbf{R}+\mathbf{S}] \mathscr{D}\left(\widehat{\mathbf{x}}_{t}\right) \\
& +2 \mathscr{D}^{\mathrm{T}}\left(\widehat{\mathbf{x}}_{t}\right) \mathbf{A}_{d} \widehat{\mathbf{x}}(t-d(t))+2 \mathscr{D}^{\mathrm{T}}\left(\widehat{\mathbf{x}}_{t}\right) \\
& \cdot(\mathbf{A}+\mathbf{B K}) \mathbf{D} \widehat{\mathbf{x}}(t-\tau)+2 \mathscr{D}^{\mathrm{T}}\left(\widehat{\mathbf{x}}_{t}\right) \\
& \cdot(\mathbf{R}+\mathbf{S}) \mathbf{D} \widehat{\mathbf{x}}(t-\tau)+\widehat{\mathbf{x}}^{\mathrm{T}}(t-\tau) \mathbf{D}^{\mathrm{T}} \\
& \cdot(\mathbf{R}+\mathbf{S}) \mathbf{D} \widehat{\mathbf{x}}(t-\tau)-(1-\mu) e^{-2 \beta d} \\
& \cdot \widehat{\mathbf{x}}^{\mathrm{T}}(t-d(t)) \mathbf{R} \widehat{\mathbf{x}}(t-d(t))-e^{-2 \beta \tau} \\
& \cdot \widehat{\mathbf{x}}^{\mathrm{T}}(t-\tau) \mathbf{S} \widehat{\mathbf{x}}(t-\tau)+2 \mathscr{D}^{\mathrm{T}}\left(\widehat{\mathbf{x}}_{t}\right) \cdot \overline{\mathbf{B}} \mathbf{L C} \mathbf{e}(t) \\
& +2 \mathscr{D}^{\mathrm{T}}\left(\mathbf{e}_{t}\right) \Delta \mathbf{A}(t) \mathbf{e}(t) \\
& +2 \mathscr{D}^{\mathrm{T}}\left(\mathbf{e}_{t}\right) \Delta \mathbf{A}_{d}(t) \mathbf{e}(t-d(t)) \\
& +2 \mathscr{D}^{\mathrm{T}}\left(\mathbf{e}_{t}\right) \Delta \mathbf{A}(t) \widehat{\mathbf{x}}(t) \\
& +2 \mathscr{D}^{\mathrm{T}}\left(\mathbf{e}_{t}\right) \Delta \mathbf{A}_{d}(t) \widehat{\mathbf{x}}(t-d(t)) \\
& -2 \beta\left[\mathbf{V}_{2}(t)+\mathbf{V}_{3}(t)+\mathbf{V}_{5}(t)+\mathbf{V}_{6}(t)\right] .
\end{aligned}
$$

Note that $\mathbf{G}=\mathbf{B}^{\mathrm{T}} \mathbf{P}$, and the Assumption 2 as well as Lemma 3 , for $\varepsilon_{i}>0(i=1, \ldots, 4)$; the following inequalities hold:

$$
\begin{aligned}
& 2 \mathscr{D}^{\mathrm{T}}\left(\mathbf{e}_{t}\right) \Delta \mathbf{A}(t) \mathbf{e}(t) \\
&= 2 \mathscr{D}^{\mathrm{T}}\left(\mathbf{e}_{t}\right) \mathbf{M} \cdot \mathbf{F}(t) \mathbf{N}\left[\mathscr{D}\left(\mathbf{e}_{t}\right)+\mathbf{D e}(t-\tau)\right] \\
& \leq \varepsilon_{1}^{-1} \mathscr{D}^{\mathrm{T}}\left(\mathbf{e}_{t}\right) \mathbf{M M}^{\mathrm{T}} \mathscr{D}\left(\mathbf{e}_{t}\right) \\
&+\varepsilon_{1}\left[\mathscr{D}\left(\mathbf{e}_{t}\right)+\mathbf{D e}(t-\tau)\right]^{\mathrm{T}} \\
& \cdot \mathbf{N}^{\mathrm{T}} \mathbf{N}\left[\mathscr{D}\left(\mathbf{e}_{t}\right)+\mathbf{D e}(t-\tau)\right], \\
& 2 \mathscr{D}^{\mathrm{T}}\left(\mathbf{e}_{t}\right) \Delta \mathbf{A}_{d}(t) \mathbf{e}(t-d(t)) \\
& \leq \varepsilon_{2}^{-1} \mathscr{D}^{\mathrm{T}}\left(\mathbf{e}_{t}\right) \mathbf{M} \mathbf{M}^{\mathrm{T}} \mathscr{D}\left(\mathbf{e}_{t}\right) \\
& \quad+\varepsilon_{2} \mathbf{e}^{\mathrm{T}}(t-d(t)) \mathbf{N}_{d}^{\mathrm{T}} \mathbf{N}_{d} \mathbf{e}(t-d(t)), \\
& 2 \mathscr{D}^{\mathrm{T}}\left(\mathbf{e}_{t}\right) \Delta \mathbf{A}(t) \widehat{\mathbf{x}}(t) \\
& \leq \varepsilon_{3}^{-1} \mathscr{D}^{\mathrm{T}}\left(\mathbf{e}_{t}\right) \mathbf{M} \mathbf{M}^{\mathrm{T}} \mathscr{D}\left(\mathbf{e}_{t}\right)+\varepsilon_{3} \widehat{\mathbf{x}}^{\mathrm{T}}(t) \mathbf{N}^{\mathrm{T}} \mathbf{N} \widehat{\mathbf{x}}(t), \\
& 2 \mathscr{D}^{\mathrm{T}}\left(\mathbf{e}_{t}\right) \Delta \mathbf{A}_{d}(t) \widehat{\mathbf{x}}(t-d(t)) \\
& \leq \varepsilon_{4}^{-1} \mathscr{D}\left(\mathbf{e}_{t}\right) \mathbf{M} \mathbf{M}^{\mathrm{T}} \mathscr{D}\left(\mathbf{e}_{t}\right) \\
& \quad+\varepsilon_{4} \widehat{\mathbf{x}}^{\mathrm{T}}(t-d(t)) \mathbf{N}_{d}^{\mathrm{T}} \mathbf{N}_{d} \widehat{\mathbf{x}}(t-d(t)) .
\end{aligned}
$$


Substituting (16) into (15) yields

$$
\begin{aligned}
& \dot{\mathbf{V}}(t)+2 \beta \mathbf{V}(t) \leq \mathscr{D}^{\mathrm{T}}\left(\mathbf{e}_{t}\right) \\
& \cdot[\operatorname{sym}\{(\mathbf{A}-\mathbf{L C})\}+\mathbf{P}+\mathbf{Q}+2 \beta \mathbf{I}] \cdot \mathscr{D}\left(\mathbf{e}_{t}\right) \\
& +2 \mathscr{D}^{\mathrm{T}}\left(\mathbf{e}_{t}\right)(\mathbf{A}-\mathbf{L C}) \operatorname{De}(t-\tau)+2 \mathscr{D}^{\mathrm{T}}\left(\mathbf{e}_{t}\right) \\
& \cdot \mathbf{A}_{d} \mathbf{e}(t-d(t))+2 \mathscr{D}^{\mathrm{T}}\left(\mathbf{e}_{t}\right)(\mathbf{P}+\mathbf{Q}) \cdot \mathbf{D e}(t-\tau) \\
& +\mathbf{e}^{\mathrm{T}}(t-\tau) \mathbf{D}^{\mathrm{T}}(\mathbf{P}+\mathbf{Q}) \mathbf{D e}(t-\tau)-e^{-2 \beta \tau} \mathbf{e}^{\mathrm{T}}(t-\tau) \\
& \text {. Qe }(t-\tau)-(1-\mu) e^{-2 \beta d} \cdot \mathbf{e}^{\mathrm{T}}(t-d(t)) \\
& \cdot \operatorname{Pe}(t-d(t))+\mathscr{D}^{\mathrm{T}}\left(\widehat{\mathbf{x}}_{t}\right) \\
& \cdot[\operatorname{sym}\{(\mathbf{A}+\mathbf{B K})\}+\mathbf{R}+\mathbf{S}+2 \beta \mathbf{I}] \mathscr{D}\left(\widehat{\mathbf{x}}_{t}\right) \\
& +2 \mathscr{D}^{\mathrm{T}}\left(\widehat{\mathbf{x}}_{t}\right) \mathbf{A}_{d} \widehat{\mathbf{x}}(t-d(t))+2 \mathscr{D}^{\mathrm{T}}\left(\widehat{\mathbf{x}}_{t}\right)(\mathbf{A}+\mathbf{B K}) \\
& \cdot \mathbf{D} \widehat{\mathbf{x}}(t-\tau)+2 \mathscr{D}^{\mathrm{T}}\left(\widehat{\mathbf{x}}_{t}\right) \cdot(\mathbf{R}+\mathbf{S}) \mathbf{D} \widehat{\mathbf{x}}(t-\tau) \\
& +\widehat{\mathbf{x}}^{\mathrm{T}}(t-\tau) \mathbf{D}^{\mathrm{T}}(\mathbf{R}+\mathbf{S}) \cdot \mathbf{D} \widehat{\mathbf{x}}(t-\tau)-(1-\mu) \\
& \cdot e^{-2 \beta d} \widehat{\mathbf{x}}^{\mathrm{T}}(t-d(t)) \mathbf{R} \cdot \widehat{\mathbf{x}}(t-d(t)) \\
& -e^{-2 \beta \tau} \widehat{\mathbf{x}}^{\mathrm{T}}(t-\tau) \mathbf{S} \widehat{\mathbf{x}}(t-\tau)+2 \mathscr{D}^{\mathrm{T}}\left(\widehat{\mathbf{x}}_{t}\right) \\
& \cdot \overline{\mathbf{B} L C}\left[\mathscr{D}\left(\mathbf{e}_{t}\right)+\mathbf{D e}(t-\tau)\right] \\
& +\left(\varepsilon_{1}^{-1}+\varepsilon_{2}^{-1}+\varepsilon_{3}^{-1}+\varepsilon_{4}^{-1}\right) \mathscr{D}^{\mathrm{T}}\left(\mathbf{e}_{t}\right) \mathbf{M M}^{\mathrm{T}} \mathscr{D}\left(\mathbf{e}_{t}\right) \\
& +\varepsilon_{1}\left[\mathscr{D}\left(\mathbf{e}_{t}\right)+\mathbf{D e}(t-\tau)\right]^{\mathrm{T}} \\
& \cdot \mathbf{N}^{\mathrm{T}} \mathbf{N}\left[\mathscr{D}\left(\mathbf{e}_{t}\right)+\mathbf{D e}(t-\tau)\right]+\varepsilon_{2} \mathbf{e}^{\mathrm{T}}(t-d(t)) \\
& \cdot \mathbf{N}_{d}^{\mathrm{T}} \mathbf{N}_{d} \mathbf{e}(t-d(t))+\varepsilon_{3} \widehat{\mathbf{x}}^{\mathrm{T}}(t) \mathbf{N}^{\mathrm{T}} \mathbf{N} \widehat{\mathbf{x}}(t) \\
& +\varepsilon_{4} \widehat{\mathbf{x}}^{\mathrm{T}}(t-d(t)) \mathbf{N}_{d}^{\mathrm{T}} \cdot \mathbf{N}_{d} \widehat{\mathbf{x}}(t-d(t)) \leq \xi^{\mathrm{T}}(t) \\
& \cdot \Theta \xi(t) \text {, }
\end{aligned}
$$

where

$$
\begin{aligned}
\boldsymbol{\xi}^{\mathrm{T}}(t) & =\left[\mathscr{D}\left(\mathbf{e}_{t}\right), \mathbf{e}(t-\tau), \mathbf{e}(t-d(t)), \mathscr{D}\left(\widehat{\mathbf{x}}_{t}\right),\right. \\
\longrightarrow & \longleftarrow \widehat{\mathbf{x}}(t-\tau), \widehat{\mathbf{x}}(t-d(t))],
\end{aligned}
$$$$
\boldsymbol{\Theta}=\left[\begin{array}{cccccc}
\boldsymbol{\Theta}_{11} & \boldsymbol{\Theta}_{12} & \mathbf{A}_{d} & (\overline{\mathbf{B}} \mathbf{L C})^{\mathrm{T}} & \mathbf{0} & \mathbf{0} \\
* & \boldsymbol{\Theta}_{22} & \mathbf{0} & (\overline{\mathbf{B}} \mathbf{L C D})^{\mathrm{T}} & \mathbf{0} & \mathbf{0} \\
* & * & \boldsymbol{\Theta}_{33} & \mathbf{0} & \mathbf{0} & \mathbf{0} \\
* & * & * & \boldsymbol{\Theta}_{44} & \boldsymbol{\Theta}_{45} & \mathbf{A}_{d} \\
* & * & * & * & \boldsymbol{\Theta}_{55} & \mathbf{0} \\
* & * & * & * & * & \boldsymbol{\Theta}_{66}
\end{array}\right],
$$

with

$$
\begin{aligned}
\boldsymbol{\Theta}_{11}= & \operatorname{sym}\{\mathbf{A}-\mathbf{L} \mathbf{C}\}+\mathbf{P}+\mathbf{Q}+2 \beta \mathbf{I} \\
& +\left(\varepsilon_{1}^{-1}+\varepsilon_{2}^{-1}+\varepsilon_{3}^{-1}+\varepsilon_{4}^{-1}\right) \mathbf{M} \mathbf{M}^{\mathrm{T}}+\varepsilon_{1} \mathbf{N}^{\mathrm{T}} \mathbf{N},
\end{aligned}
$$

$$
\begin{aligned}
& \boldsymbol{\Theta}_{12}=(\mathbf{A}-\mathbf{L} \mathbf{C}) \mathbf{D}+(\mathbf{P}+\mathbf{Q}) \mathbf{D}+\varepsilon_{1} \mathbf{N}^{\mathrm{T}} \mathbf{N D}, \\
& \boldsymbol{\Theta}_{22}=\mathbf{D}^{\mathrm{T}}(\mathbf{P}+\mathbf{Q}) \mathbf{D}-e^{-2 \beta \tau} \mathbf{Q}+\varepsilon_{1}(\mathbf{N D})^{\mathrm{T}} \mathbf{N D}, \\
& \boldsymbol{\Theta}_{33}=-(1-\mu) e^{-2 \beta d} \mathbf{P}+\varepsilon_{2} \mathbf{N}_{d}^{\mathrm{T}} \mathbf{N}_{d}, \\
& \boldsymbol{\Theta}_{44}=\operatorname{sym}\{\mathbf{A}+\mathbf{B K}\}+\mathbf{R}+\mathbf{S}+2 \beta \mathbf{I}+\varepsilon_{3} \mathbf{N}^{\mathrm{T}} \mathbf{N}, \\
& \boldsymbol{\Theta}_{45}=(\mathbf{A}+\mathbf{B K}) \mathbf{D}+(\mathbf{R}+\mathbf{S}) \mathbf{D}+\varepsilon_{3} \mathbf{N}^{\mathrm{T}} \mathbf{N D}, \\
& \boldsymbol{\Theta}_{55}=-e^{-2 \beta \tau} \mathbf{S}+\mathbf{D}^{\mathrm{T}}(\mathbf{R}+\mathbf{S}) \mathbf{D}+\varepsilon_{3}(\mathbf{N D})^{\mathrm{T}} \mathbf{N D}, \\
& \boldsymbol{\Theta}_{66}=-(1-\mu) e^{-2 \beta d} \mathbf{R}+\varepsilon_{4} \mathbf{N}_{d}^{\mathrm{T}} \mathbf{N}_{d} .
\end{aligned}
$$

It can be shown that if the LMI (9) is satisfied, $\boldsymbol{\Theta}<\mathbf{0}$ can be held by using the well-known Schur complement formula. Then, we get

$$
\dot{\mathbf{V}}(t)+2 \beta \mathbf{V}(t) \leq \boldsymbol{\xi}^{\mathrm{T}}(t) \Theta \boldsymbol{\xi}(t)<0 \quad(\text { for } \boldsymbol{\xi}(t) \neq \mathbf{0}),
$$

which means that the overall closed-loop system is robust asymptotically stable, and it is followed by $\widehat{\mathbf{x}}(t) \rightarrow \mathbf{0}, \mathbf{e}(t) \rightarrow$ $\mathbf{0}$ as $t \rightarrow+\infty$.

Next, when $\mathbf{w}(t) \neq \mathbf{0}$, assume zero initial conditions for the overall system. Consider the following inequality:

$$
\begin{aligned}
\dot{\mathbf{V}}(t) & +2 \beta \mathbf{V}(t)-2 \mathbf{w}^{\mathrm{T}}(t) \mathbf{z}_{e}(t)-\gamma \mathbf{w}^{\mathrm{T}}(t) \mathbf{w}(t) \\
\leq & \boldsymbol{\eta}^{\mathrm{T}}(t) \widehat{\boldsymbol{\Theta}} \boldsymbol{\eta}(t),
\end{aligned}
$$

where

$$
\boldsymbol{\eta}^{\mathrm{T}}(t)=\left[\boldsymbol{\xi}^{\mathrm{T}}(t), \mathbf{w}(t)\right],
$$

$\widehat{\Theta}$

$$
=\left[\begin{array}{ccccccc}
\boldsymbol{\Theta}_{11} & \boldsymbol{\Theta}_{12} & \mathbf{A}_{d} & (\overline{\mathbf{B}} \mathbf{L C})^{\mathrm{T}} & \mathbf{0} & \mathbf{0} & -\mathbf{C}^{\mathrm{T}}+\mathbf{G}_{w} \\
* & \boldsymbol{\Theta}_{22} & \mathbf{0} & (\overline{\mathbf{B}} \mathbf{L C D})^{\mathrm{T}} & \mathbf{0} & \mathbf{0} & -(\mathbf{C D})^{\mathrm{T}} \\
* & * & \boldsymbol{\Theta}_{33} & \mathbf{0} & \mathbf{0} & \mathbf{0} & \mathbf{0} \\
* & * & * & \boldsymbol{\Theta}_{44} & \boldsymbol{\Theta}_{45} & \mathbf{A}_{d} & \mathbf{0} \\
* & * & * & * & \boldsymbol{\Theta}_{55} & \mathbf{0} & \mathbf{0} \\
* & * & * & * & * & \boldsymbol{\Theta}_{66} & \mathbf{0} \\
* & * & * & * & * & * & -\gamma \mathbf{I}
\end{array}\right] .
$$

Thus, by invoking the Schur complement and applying (9), it is clear that

$$
\dot{\mathbf{V}}(t)-2 \mathbf{w}^{\mathrm{T}}(t) \mathbf{z}_{e}(t)-\gamma \mathbf{w}^{\mathrm{T}}(t) \mathbf{w}(t) \leq 0 .
$$

Integrating both sides of the above inequality with respect to $t$ over the time period $[0, T]$, we get

$$
\begin{gathered}
\mathbf{V}(T)-\mathbf{V}(0)-2 \int_{0}^{T} \mathbf{w}^{T}(t) \mathbf{z}_{e}(t) \mathrm{d} t \\
-\gamma \int_{0}^{T} \mathbf{w}^{\mathrm{T}}(t) \mathbf{w}(t) \mathrm{d} t \leq 0
\end{gathered}
$$


Under the zero initial conditions, we have $\mathbf{V}(0)=0$ and $\mathbf{V}(T) \geq 0$, so the above inequality guarantees (8). Hence, the overall closed-loop system with (4) and (7) is said to be robust asymptotically stable and passive. The proof is completed.

Remark 7. If matrix $\mathbf{D} \equiv \mathbf{0}$, then the neutral-type system under consideration becomes a retarded system. For this retarded system, the state estimate dynamic system and error dynamic system have the same form as (3) and (4) with $\mathrm{D} \equiv \mathbf{0}$, respectively. So, based upon Theorem 6 , we have the following result for the retarded system.

Corollary 8. The overall closed-loop retarded system (i.e., system (4) and (7) with $\mathbf{D} \equiv \mathbf{0}$ ) is robust exponentially stable and passive, if there exist symmetric positive definite matrices $\mathbf{P}>\mathbf{0}, \mathbf{R}>\mathbf{0}$, matrices $\mathbf{L}, \mathbf{K}$, and scalars $\varepsilon_{i}>0(i=1, \ldots, 4)$ satisfying the following linear matrix inequality

$$
\left[\begin{array}{cccccc}
\boldsymbol{\Sigma}_{11} & \mathbf{A}_{d} & (\overline{\mathbf{B}} \mathbf{L C})^{\mathrm{T}} & \mathbf{0} & -\mathbf{C}^{\mathrm{T}}+\mathbf{G}_{w} & \mathbf{J}_{3} \\
* & \boldsymbol{\Sigma}_{22} & \mathbf{0} & \mathbf{0} & \mathbf{0} & \mathbf{0} \\
* & * & \boldsymbol{\Sigma}_{33} & \mathbf{A}_{d} & \mathbf{0} & \mathbf{0} \\
* & * & * & \boldsymbol{\Sigma}_{44} & \mathbf{0} & \mathbf{0} \\
* & * & * & * & -\gamma \mathbf{I} & \mathbf{0} \\
* & * & * & * & * & -\mathbf{J}_{4}
\end{array}\right]<\mathbf{0},
$$

where

$$
\begin{aligned}
\boldsymbol{\Sigma}_{11} & =\operatorname{sym}\{\mathbf{A}-\mathbf{L C}\}+\mathbf{P}+2 \beta \mathbf{I}+\varepsilon_{1} \mathbf{N}^{\mathrm{T}} \mathbf{N}, \\
\boldsymbol{\Sigma}_{22} & =-e^{-2 \beta d} \mathbf{P}+\varepsilon_{3} \mathbf{N}_{d}^{\mathrm{T}} \mathbf{N}_{d}, \\
\boldsymbol{\Sigma}_{33} & =\operatorname{sym}\{\mathbf{A}+\mathbf{B K}\}+\mathbf{R}+2 \beta \mathbf{I}+\varepsilon_{2} \mathbf{N}^{\mathrm{T}} \mathbf{N}, \\
\boldsymbol{\Sigma}_{44} & =-e^{-2 \beta d} \mathbf{R}+\varepsilon_{4} \mathbf{N}_{d}^{\mathrm{T}} \mathbf{N}_{d}, \\
\mathbf{J}_{3} & =\left[\begin{array}{lll}
\mathbf{M} & \mathbf{M} \mathbf{M} \quad \mathbf{M}
\end{array}\right], \\
\mathbf{J}_{4} & =\operatorname{diag}\left\{\varepsilon_{1} \mathbf{I}, \varepsilon_{2} \mathbf{I}, \varepsilon_{3} \mathbf{I}, \varepsilon_{4} \mathbf{I}\right\} .
\end{aligned}
$$

The desired result can be carried out by employing the same technique used as those in Theorem 6, and the proof is omitted here.

Remark 9. A simple stability criterion for the uncertain closed-loop system is given, which is both a delaydependent and delay-derivative dependent condition. Thus, it is expected to be less conservative than the result discussed without time delay in the literature [28].

3.3. Reachability Analysis. In the following part, by means of the state estimate in (3), a SM controller will be synthesized to ensure the reachability of the sliding surface $\mathbf{s}(t, \widehat{\mathbf{x}})=\mathbf{0}$.

To this end, the following SM controller is designed

$$
\mathbf{u}_{v s}(t)=\mathbf{K} \widehat{\mathbf{x}}(t)-\rho(t, \widehat{\mathbf{x}}) \operatorname{sgn}(\mathbf{s}(t, \widehat{\mathbf{x}}))
$$

where

$$
\begin{aligned}
\rho(t, \widehat{\mathbf{x}})= & \alpha+\left\|(\mathbf{G B})^{-1} \mathbf{G L z}(t)\right\| \\
& +\left\|(\mathbf{G B})^{-1} \mathbf{G L C} \widehat{\mathbf{x}}(t)\right\|,
\end{aligned}
$$

$\alpha>0$ is a small constant, and the matrix $\mathbf{K}$ is given in Theorem 6.

In the following theorem, we will analyze the reachability of the sliding surface $\mathbf{s}(t, \widehat{\mathbf{x}})=\mathbf{0}$.

Theorem 10. Consider the state observer system (3). If the control input $\mathbf{u}(t)$ is chosen as (28), then the trajectory of the observer system (3) can converge to the sliding surface $\mathbf{s}(t, \widehat{\mathbf{x}})=$ $\mathbf{0}$ in a finite moment.

Proof. Choose the Lyapunov function as

$$
\widehat{\mathbf{V}}(t)=0.5 \mathbf{s}(t, \widehat{\mathbf{x}})(\mathbf{G B})^{-1} \mathbf{s}(t, \widehat{\mathbf{x}})
$$

Thus, if follows from (5) that

$$
\begin{aligned}
& \dot{\widehat{\mathbf{V}}}(t)=\mathbf{s}(t, \widehat{\mathbf{x}})(\mathbf{G B})^{-1} \dot{\mathbf{s}}(t, \widehat{\mathbf{x}})=\mathbf{s}(t, \widehat{\mathbf{x}})(\mathbf{G B})^{-1} \\
& \cdot\{\mathbf{G}[\dot{\widehat{\mathbf{x}}}(t)-\dot{\widehat{\mathbf{x}}}(t-\tau)] \\
& \left.-\mathbf{G}(\mathbf{A}+\mathbf{B K}) \widehat{\mathbf{x}}(t)-\mathbf{G} \mathbf{A}_{d} \widehat{\mathbf{x}}(t-d(t))\right\}=\mathbf{s}(t, \widehat{\mathbf{x}}) \\
& \cdot(\mathbf{G B})^{-1}\{\mathbf{G B u}(t)+\mathbf{G L}[\mathbf{z}(t)-\mathbf{C} \widehat{\mathbf{x}}(t)] \\
& -\mathbf{G B K} \widehat{\mathbf{x}}(t)\}=\mathbf{s}(t, \widehat{\mathbf{x}})\{\mathbf{u}(t) \\
& \left.+(\mathbf{G B})^{-1} \mathbf{G L}[\mathbf{z}(t)-\mathbf{C} \widehat{\mathbf{x}}(t)]-\mathbf{K} \widehat{\mathbf{x}}(t)\right\}=\mathbf{s}(t, \widehat{\mathbf{x}}) \\
& \cdot\left\{(\mathbf{G B})^{-1} \mathbf{G L}[\mathbf{z}(t)-\mathbf{C} \widehat{\mathbf{x}}(t)]\right. \\
& -\rho(t, \widehat{\mathbf{x}}) \operatorname{sgn}(\mathbf{s}(t, \widehat{\mathbf{x}}(t)))\} \leq-\alpha\|\mathbf{s}(t, \widehat{\mathbf{x}})\|<0, \\
& \quad \text { if } \mathbf{s}(t, \widehat{\mathbf{x}}) \neq \mathbf{0} .
\end{aligned}
$$

Meanwhile, note that

$$
\begin{aligned}
2 \widehat{\mathbf{V}}(t) & =\mathbf{s}^{\mathrm{T}}(t, \mathbf{x})(\mathbf{G B})^{-1} \mathbf{s}(t, \mathbf{x}) \\
& \leq \lambda_{\max }\left((\mathbf{G B})^{-1}\right) \cdot\|\mathbf{s}(t, \mathbf{x})\|^{2} .
\end{aligned}
$$

It is clear that

$$
\dot{\widehat{\mathbf{V}}}(t) \leq-\widehat{\alpha} \cdot \widehat{\mathbf{V}}^{1 / 2}(t),
$$

where $\widehat{\alpha}=\alpha \sqrt{2 \lambda_{\max }\left((\mathbf{G B})^{-1}\right)^{-1}}$.

Therefore, the existence of an instant $T_{\text {fin }}$ can be estimated as $T_{\text {fin }} \leq \sqrt{\widehat{\mathbf{V}}(0)} / \widehat{\alpha}$ such that $\widehat{\mathbf{V}}(t)=0$ (equivalently, $\mathbf{s}(t, \widehat{\mathbf{x}})=$ 0) when $t \geq T_{\text {fin }}$. Thus, the state of the observer system (3) can be driven onto the predefined sliding surface $\mathbf{s}(t, \widehat{\mathbf{x}})=\mathbf{0}$ in finite time, thereby completing the proof. 
TABLE 1: The feasibility and observer gain $L$ when $\beta=0.0836$.

\begin{tabular}{lcccc}
\hline Method & Time delay d & Feasible solution & Observer gain $L$ \\
\hline $\begin{array}{l}\text { Reference [28] } \\
\text { Theorem 6 }\end{array}$ & 3.5 & No & - \\
\hline $\begin{array}{l}\text { Reference [28] } \\
\text { Theorem 6 }\end{array}$ & 6.5 & No & {$\left[\begin{array}{llll}-1.3468 & -0.5238 & 0.7936\end{array}\right]^{T}$} \\
\hline
\end{tabular}

TABLE 2: Upper delay bound $d$ for various $\mu$.

\begin{tabular}{|c|c|c|c|c|c|c|c|}
\hline Method & Condition & $\tau=1.1$ & $\tau=1.2$ & $\tau=1.3$ & $\tau=1.4$ & $\tau=1.5$ & $\tau=1.6$ \\
\hline Reference [28] & \multirow{2}{*}{$\mu=0.2$} & \multicolumn{6}{|c|}{ No results } \\
\hline Theorem 6 & & 3.5985 & 3.0835 & 2.4854 & 1.7759 & 0.9095 & No \\
\hline Reference [28] & $u=05$ & \multicolumn{6}{|c|}{ No results } \\
\hline Theorem 6 & & 2.0318 & 1.5168 & 0.9187 & 0.2092 & No & No \\
\hline
\end{tabular}

\section{Numerical Example}

Consider an example of the system with the following parameters:

$$
\begin{aligned}
& \mathbf{A}=\left[\begin{array}{ccc}
-5.0 & 2.0 & 0.0 \\
1.0 & -4.0 & -1.0 \\
-3.0 & 1.5 & -5.0
\end{array}\right] \\
& \mathbf{A}_{d}=\left[\begin{array}{ccc}
-0.2 & 0.1 & 0.3 \\
0.0 & -0.1 & -0.4 \\
1.0 & -0.2 & -0.1
\end{array}\right] \text {, } \\
& \mathbf{D}=\left[\begin{array}{ccc}
-0.2 & 0.0 & 0.0 \\
-0.2 & 0.2 & 0.4 \\
0.0 & 0.2 & -0.1
\end{array}\right] \text {, } \\
& \mathbf{B}=\left[\begin{array}{c}
-2 \\
1.5 \\
2
\end{array}\right] \text {, } \\
& \mathbf{C}=\left[\begin{array}{c}
-1.5 \\
-2.0 \\
1.0
\end{array}\right]^{\mathrm{T}} \\
& \mathbf{M}=\left[\begin{array}{ccc}
0.1 & 0.0 & 0.0 \\
0.0 & -0.1 & 0.0 \\
-0.2 & 0.0 & 0.2
\end{array}\right] \text {, } \\
& \mathbf{N}=\left[\begin{array}{ccc}
0.1 & 0.0 & 0.0 \\
0.0 & 0.0 & -0.1 \\
0.0 & -0.1 & 0.0
\end{array}\right] \text {, } \\
& \mathbf{N}_{d}=\left[\begin{array}{ccc}
0.1 & 0.0 & -0.2 \\
0.0 & -0.1 & 0.0 \\
0.0 & 0.0 & -0.1
\end{array}\right] \text {, }
\end{aligned}
$$

$$
\mathbf{F}(t)=\left[\begin{array}{ccc}
\sin t & 0 & 0 \\
0 & \sin t & 0 \\
0 & 0 & \sin t
\end{array}\right],
$$$$
\mathbf{G}_{w}=\left[\begin{array}{c}
0.2 \\
0.4 \\
0.5
\end{array}\right] \text {. }
$$

In addition, the constant neutral-term delay $\tau=1.2$ and the time-varying delay $d(t)$ is chosen as $d(t)=0.3+0.2 \sin t$ such that $\mu=0.2$ and $d=0.5$ can be given. Select $\beta=0.15$, the performance index $\gamma=1.4012$, and the external disturbance $\mathbf{w}(t)=1 /\left(1+e^{t}\right)$.

Our aims here are as follows: (1) to show the superiority of Theorem 6 when compared with the relative result in the literature [28]; (2) to solve the gain matrix $\mathbf{K}$ and L in Theorem 6 such that the overall closed-loop system composed of (4) and (7) can be robust asymptotically stable and passive; (3) to design a SMC law given in (28) such that state of the observer system (3) can be driven onto the sliding surface $\mathbf{s}(t, \widehat{\mathbf{x}})=\mathbf{0}$ in finite time.

Firstly, Tables 1 and 2 give a detailed comparison of results on the feasible solution and maximum allowed delay bound via the method in [28] and Theorem 6 in this paper, respectively.

In terms of conservatism, the results in the tables clearly show that the condition in this paper outperforms that in [28].

Secondly, by solving the LMI (9), we obtain the parameters as follows:

$$
\begin{aligned}
& \mathbf{P}=\left[\begin{array}{ccc}
3.1523 & -0.9756 & 1.5372 \\
-0.9756 & 0.6376 & -0.1278 \\
1.5372 & -0.1278 & 1.7957
\end{array}\right], \\
& \mathbf{Q}=\left[\begin{array}{ccc}
4.1726 & -1.1968 & 0.1225 \\
-1.1968 & 1.2350 & 0.1274 \\
0.1225 & 0.1274 & 3.5446
\end{array}\right],
\end{aligned}
$$




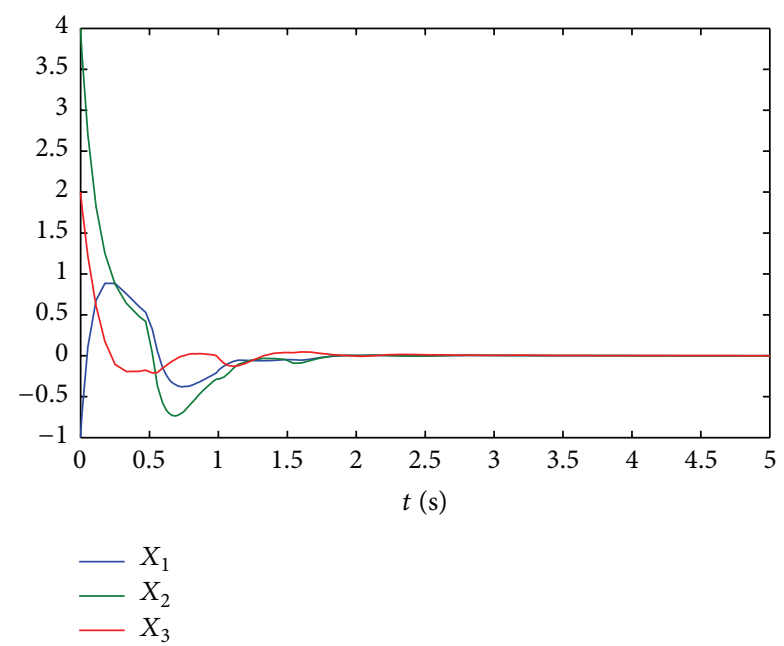

FIgURE 1: Trajectories of state $\mathbf{x}(t)$.

$$
\begin{aligned}
& \mathbf{R}=\left[\begin{array}{ccc}
4.8403 & -2.8910 & 0.6796 \\
-2.8910 & 2.2971 & -0.2332 \\
0.6796 & -0.2332 & 1.6090
\end{array}\right], \\
& \mathbf{S}=\left[\begin{array}{ccc}
4.9254 & -4.0778 & -1.3562 \\
-4.0778 & 4.8071 & 2.7366 \\
-1.3562 & 2.7366 & 6.2353
\end{array}\right], \\
& \varepsilon_{1}=4.8543 \\
& \varepsilon_{2}=1.5221, \\
& \varepsilon_{3}=13.8840, \\
& \varepsilon_{4}=6.6893 .
\end{aligned}
$$

And the gain matrices are

$$
\begin{aligned}
\mathbf{L} & =\left[\begin{array}{lll}
-0.9433 & -0.1601 & 0.6282
\end{array}\right]^{\mathrm{T}}, \\
\mathbf{K} & =\left[\begin{array}{lll}
2.5941 & -1.9850 & -1.1917
\end{array}\right]
\end{aligned}
$$

Thirdly, design of the SMC law will be taken into account. Let the adjustable parameter $\alpha$ be $\alpha=0.60$. Thus, the proposed SMC law becomes

$$
\begin{aligned}
& u_{v s}(t)=\left[\begin{array}{lll}
2.5941 & -1.9850 & -1.1917
\end{array}\right] \widehat{\mathbf{x}}(t)-[0.60 \\
& \quad+\|0.3326 \cdot \mathbf{z}(t)\| \\
& \left.+\left\|\left[\begin{array}{lll}
-0.4988 & -0.6651 & 0.3326
\end{array}\right] \widehat{\mathbf{x}}(t)\right\|\right] \\
& \quad \cdot \operatorname{sgn}(s(t, \widehat{\mathbf{x}}))
\end{aligned}
$$

For the sake of avoiding the chattering problem, the unit vector term in the SMC law could be replaced by $s(t, \widehat{\mathbf{x}})[\|s(t, \widehat{\mathbf{x}})\|+0.05]^{-1}$. The initial conditions are chosen as $\boldsymbol{\varphi}(t)=\left[\begin{array}{lll}-1 & 4 & 2\end{array}\right]^{\mathrm{T}}$ and $\hat{\boldsymbol{\varphi}}(t)=\left[\begin{array}{lll}-1 & 3 & 4\end{array}\right]^{\mathrm{T}}, t \in[-1.2,0]$.

Simulation results are provided in Figures 1-4.

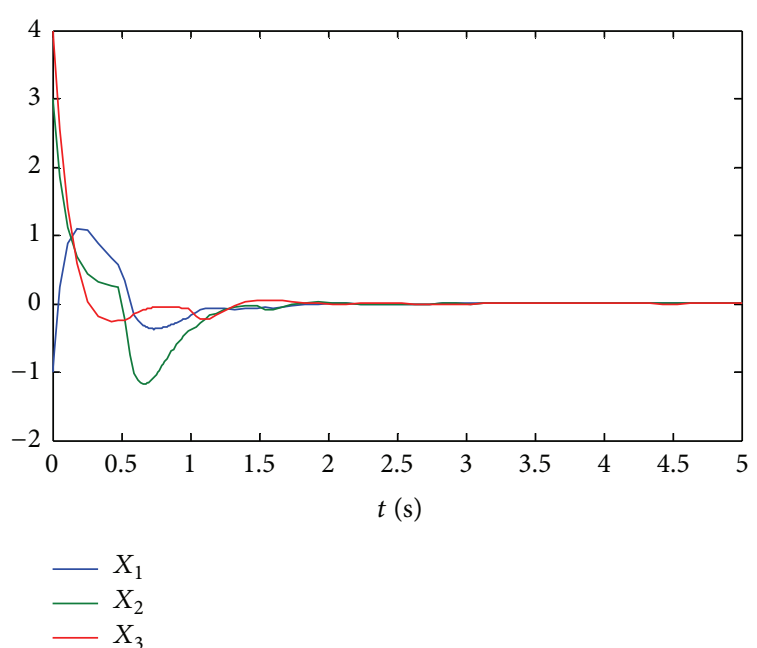

Figure 2: Trajectories of state estimate $\widehat{\mathbf{x}}(t)$.

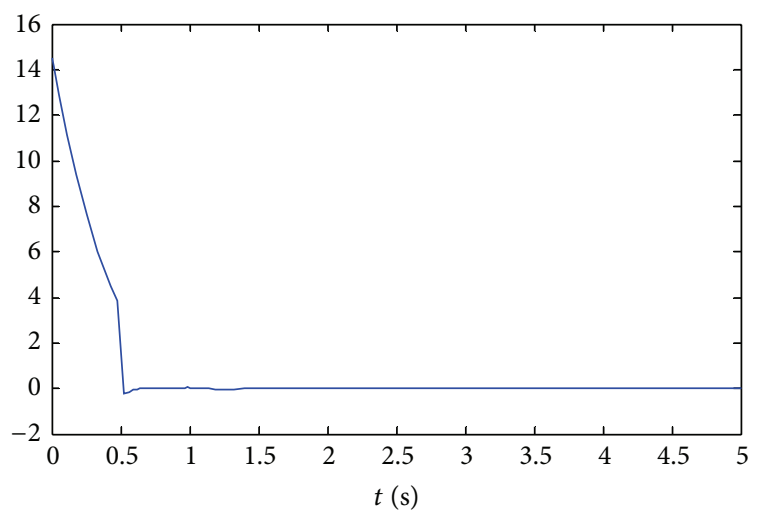

FIgURE 3: Trajectory of sliding functional $\mathbf{s}(t, \widehat{\mathbf{x}})$.

The state response of the neutral system is plotted in Figure 1. Meanwhile, the state response of the observer is shown in Figure 2. From Figures 1 and 2, it can be seen that the state $\mathbf{x}(t)$ and its estimate $\widehat{\mathbf{x}}(t)$ have a good convergent performance even with the external disturbance. Figure 3 indicates the curve of the sliding functional $s(t, \widehat{\mathbf{x}})$. From Figure 3, it can be seen that the sliding mode is attained in a finite moment approximated to $t_{\text {fin }} \approx 0.6 \mathrm{~s}$. After that time, response of the state estimate is globally robust asymptotically stable, which results in the passivity and robust asymptotic stability of the overall closed-loop system.

The SMC law $u_{v_{s}}(t)$ has played an important role in driving the state $\widehat{\mathbf{x}}(t)$ onto the sliding surface $\mathbf{s}(t, \widehat{\mathbf{x}})=\mathbf{0}$, which is depicted in Figure 4.

\section{Conclusion}

In this paper, the problem of observer-based ISMC with passivity has been studied for a class of uncertainty NTSTD. The state observer has been designed to generate the estimate of system state, based on which a SMC technique has been developed to stabilize the resulting closed-loop system. A 


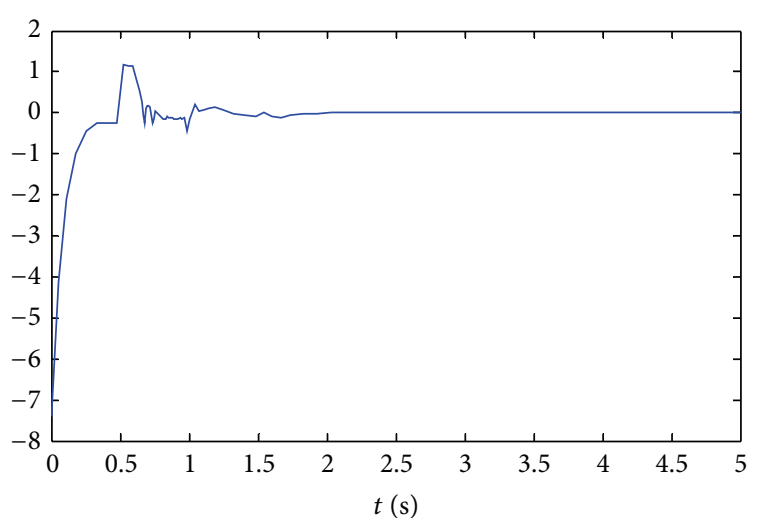

Figure 4: Control input $\mathbf{u}(t)$.

sufficient condition of the passivity and robust asymptotic stability has been derived for the overall closed-loop system composed of the observer SM dynamic system and the state error dynamic system via LMI. The proposed SMC law can guarantee the reachability of the sliding surface in state estimate space in finite time. Finally, a numerical example has been given to illustrate the superiority and effectiveness of the obtained results. We will consider the observer-based ISMC for uncertain nonlinear NTSTD in the next paper.

\section{Conflict of Interests}

The authors declare that there is no conflict of interests regarding the publication of this paper.

\section{Acknowledgment}

This work is partially supported by National Nature Science Foundation of China (Grant no. 61374079).

\section{References}

[1] P. Hippe and J. Deutscher, Design of Observer-Based Compensators, Springer, London, UK, 2009.

[2] S. He, "Non-fragile passive controller design for nonlinear Markovian jumping systems via observer-based controls," Neurocomputing, vol. 147, pp. 350-357, 2015.

[3] Y. Zhang, P. Shi, and S. K. Nguang, "Observer-based finite-time $H_{\infty}$ control for discrete singular stochastic systems," Applied Mathematics Letters, vol. 38, pp. 115-121, 2014.

[4] S. Ibrir, "Observer-based control of a class of time-delay nonlinear systems having triangular structure," Automatica, vol. 47, no. 2, pp. 388-394, 2011.

[5] M. Darouach, "Reduced-order observers for linear neutral delay systems," IEEE Transactions on Automatic Control, vol. 50, no. 9, pp. 1407-1413, 2005.

[6] Z. Wang, Y. Shen, X. Zhang, and Q. Wang, "Observer design for discrete-time descriptor systems: an LMI approach," Systems \& Control Letters, vol. 61, no. 6, pp. 683-687, 2012.

[7] Y. Niu, J. Lam, X. Wang, and D. W. Ho, "Observer-based sliding mode control for nonlinear state-delayed systems," International Journal of Systems Science, vol. 35, no. 2, pp. 139150, 2004.
[8] X.-G. Yan, S. K. Spurgeon, and C. Edwards, "Sliding mode control for time-varying delayed systems based on a reducedorder observer," Automatica, vol. 46, no. 8, pp. 1354-1362, 2010.

[9] L. Liu, Z. Han, and W. Li, " $H_{\infty}$ non-fragile observer-based sliding mode control for uncertain time-delay systems," Journal of the Franklin Institute, vol. 347, no. 2, pp. 567-576, 2010.

[10] H. Yang, Y. Xia, and P. Shi, "Observer-based sliding mode control for a class of discrete systems via delta operator approach," Journal of the Franklin Institute, vol. 347, no. 7, pp. 1199-1213, 2010.

[11] L. Wu, P. Shi, and H. Gao, "State estimation and slidingmode control of Markovian jump singular systems," IEEE Transactions on Automatic Control, vol. 55, no. 5, pp. 1213-1219, 2010.

[12] L. Gao, D. Wang, and Y. Wu, "Non-fragile observer-based sliding mode control for Markovian jump systems with mixed mode-dependent time delays and input nonlinearity," Applied Mathematics and Computation, vol. 229, pp. 374-395, 2014.

[13] F. Li, L. Wu, P. Shi, and C.-C. Lim, "State estimation and sliding mode control for semi-Markovian jump systems with mismatched uncertainties," Automatica, vol. 51, pp. 385-393, 2015.

[14] M. Liu and G. Sun, "Observer-based sliding mode control for Itô stochastic time-delay systems with limited capacity channel," Journal of the Franklin Institute. Engineering and Applied Mathematics, vol. 349, no. 4, pp. 1602-1616, 2012.

[15] M. Mahmoud, Robust Control and Filtering for Time-Delay Systems, Marcel Decker, New York, NY, USA, 2000.

[16] Y. He, M. Wu, J.-H. She, and G.-P. Liu, "Delay-dependent robust stability criteria for uncertain neutral systems with mixed delays," Systems and Control Letters, vol. 51, no. 1, pp. 57-65, 2004.

[17] J. H. Park, "Novel robust stability criterion for a class of neutral systems with mixed delays and nonlinear perturbations," Applied Mathematics and Computation, vol. 161, no. 2, pp. 413421, 2005.

[18] J. H. Park, O. Kwon, and S. Won, "LMI approach to robust $H_{\infty}$ filtering for neutral delay differential systems," Applied Mathematics and Computation, vol. 150, no. 1, pp. 235-244, 2004.

[19] S. Lakshmanan, T. Senthilkumar, and P. Balasubramaniam, "Improved results on robust stability of neutral systems with mixed time-varying delays and nonlinear perturbations," Applied Mathematical Modelling, vol. 35, no. 11, pp. 5355-5368, 2011.

[20] Y. Sun, "On simple stability criteria for nonlinear neutral systems with multiple time delays," Applied Mathematics Letters, vol. 25, no. 11, pp. 1911-1915, 2012.

[21] R. Lu, H. Wu, and J. Bai, "New delay-dependent robust stability criteria for uncertain neutral systems with mixed delays," Journal of the Franklin Institute, vol. 351, no. 3, pp. 1386-1399, 2014.

[22] U. Baser and B. Kizilsac, "Dynamic output feedback $H_{\infty}$ control problem for linear neutral systems," IEEE Transactions on Automatic Control, vol. 52, no. 6, pp. 1113-1118, 2007.

[23] S. Arik, "An analysis of stability of neutral-type neural systems with constant time delays," Journal of the Franklin Institute, vol. 351, no. 11, pp. 4949-4959, 2014.

[24] Y. Niu, T. Jia, J. Huang, and J. Liu, "Design of sliding mode control for neutral delay systems with perturbation in control channels," Optimal Control Applications \& Methods, vol. 33, no. 3, pp. 363-374, 2012. 
[25] Y. Niu, J. Lam, and X. Wang, "Sliding-mode control for uncertain neutral-type systems with time-delay," IEE Proceedings: Control Theory and Applications, vol. 151, no. 1, pp. 38-44, 2004.

[26] Y. Niu, J. Lam, X. Wang, and D. W. C. Ho, "Neural adaptive sliding mode control for a class of nonlinear neutral delay systems," Journal of Dynamic Systems, Measurement and Control, Transactions of the ASME, vol. 130, no. 6, pp. 1101-1107, 2008.

[27] C. Gao, Z. Liu, and R. Xu, "On exponential stabilization for a class of neutral-type systems with parameter uncertainties: an integral sliding mode approach," Applied Mathematics and Computation, vol. 219, no. 23, pp. 11044-11055, 2013.

[28] L. Wu, C. Wang, and Q. Zeng, "Observer-based sliding mode control for a class of uncertain nonlinear neutral delay systems," Journal of the Franklin Institute, vol. 345, no. 3, pp. 233-253, 2008. 

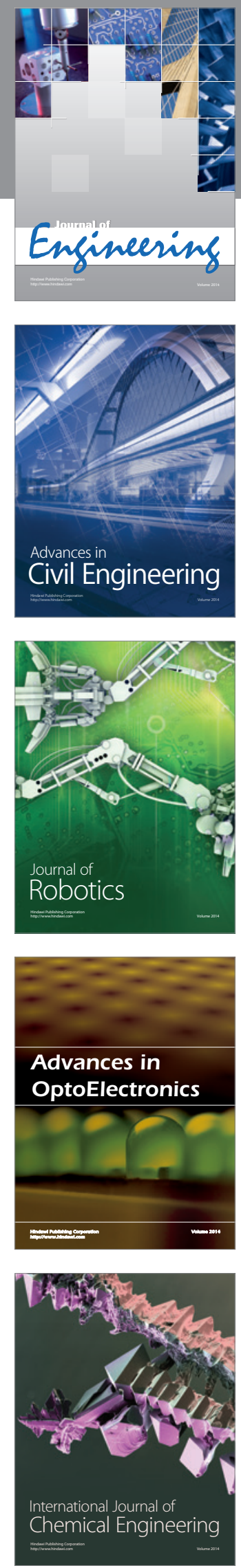

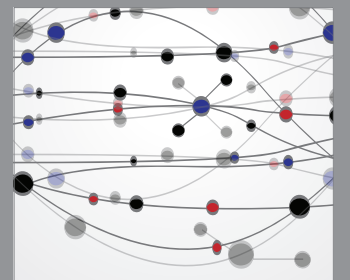

The Scientific World Journal
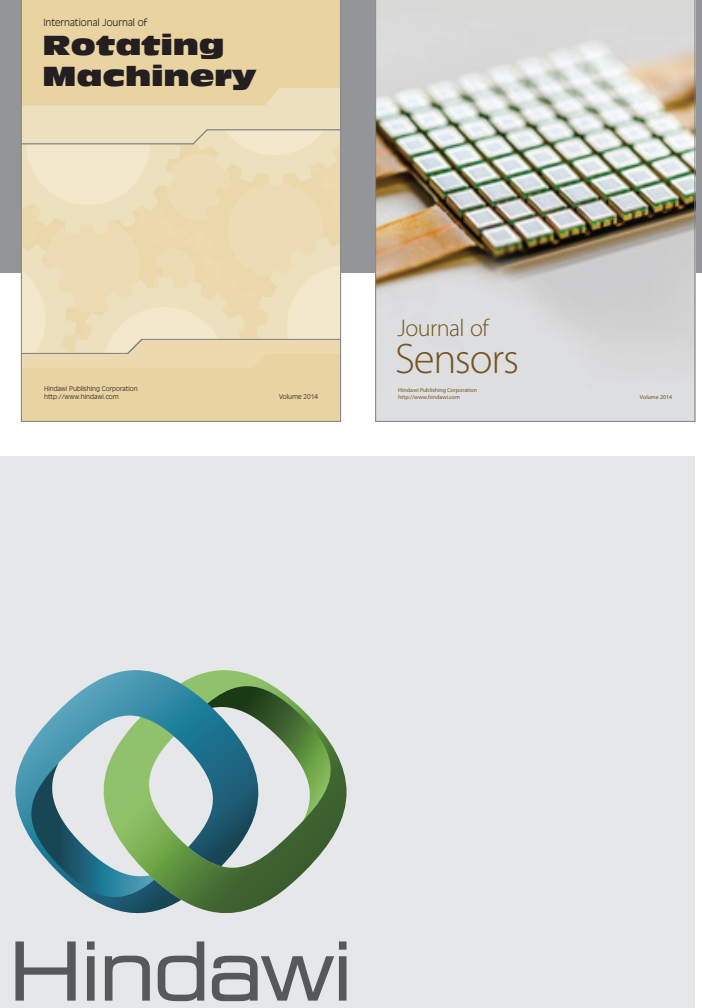

Submit your manuscripts at http://www.hindawi.com
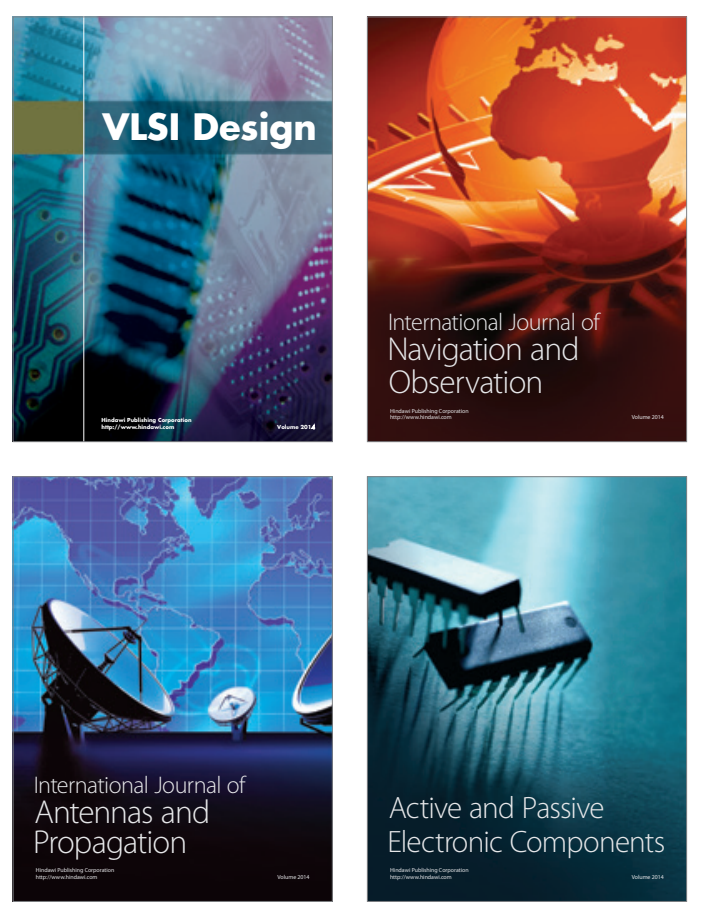
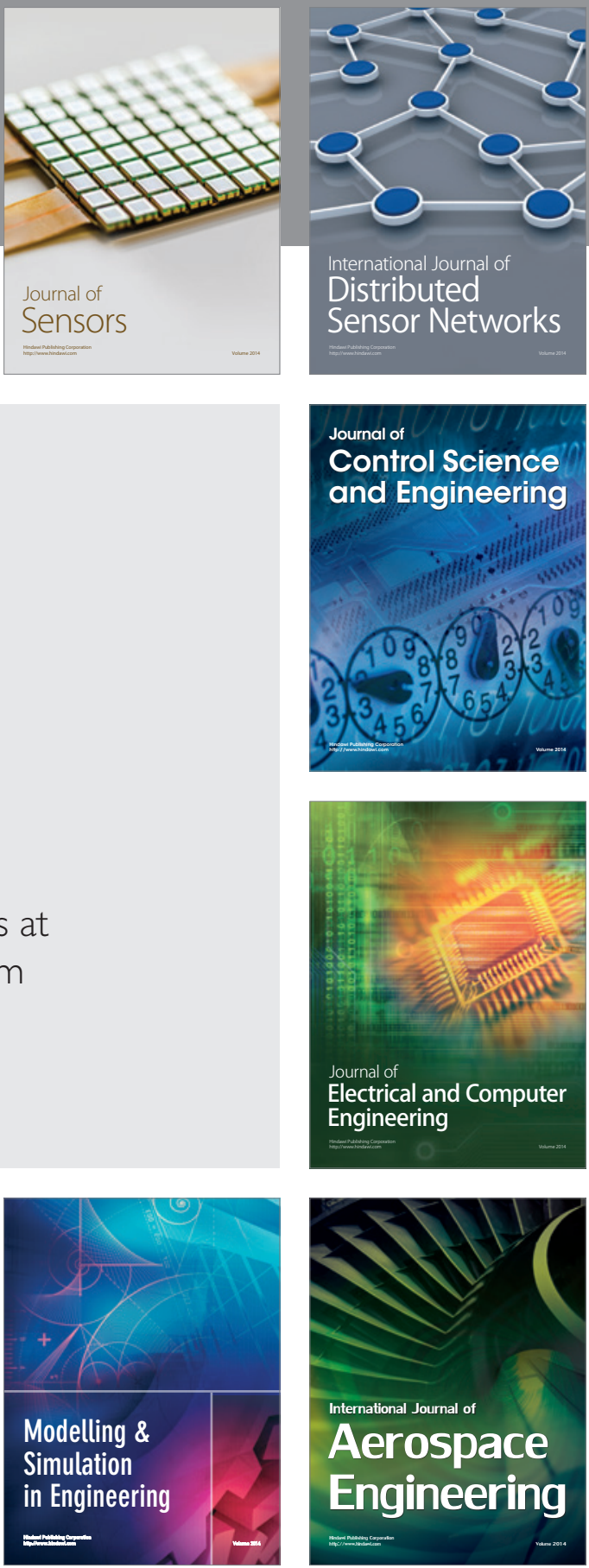

Journal of

Control Science

and Engineering
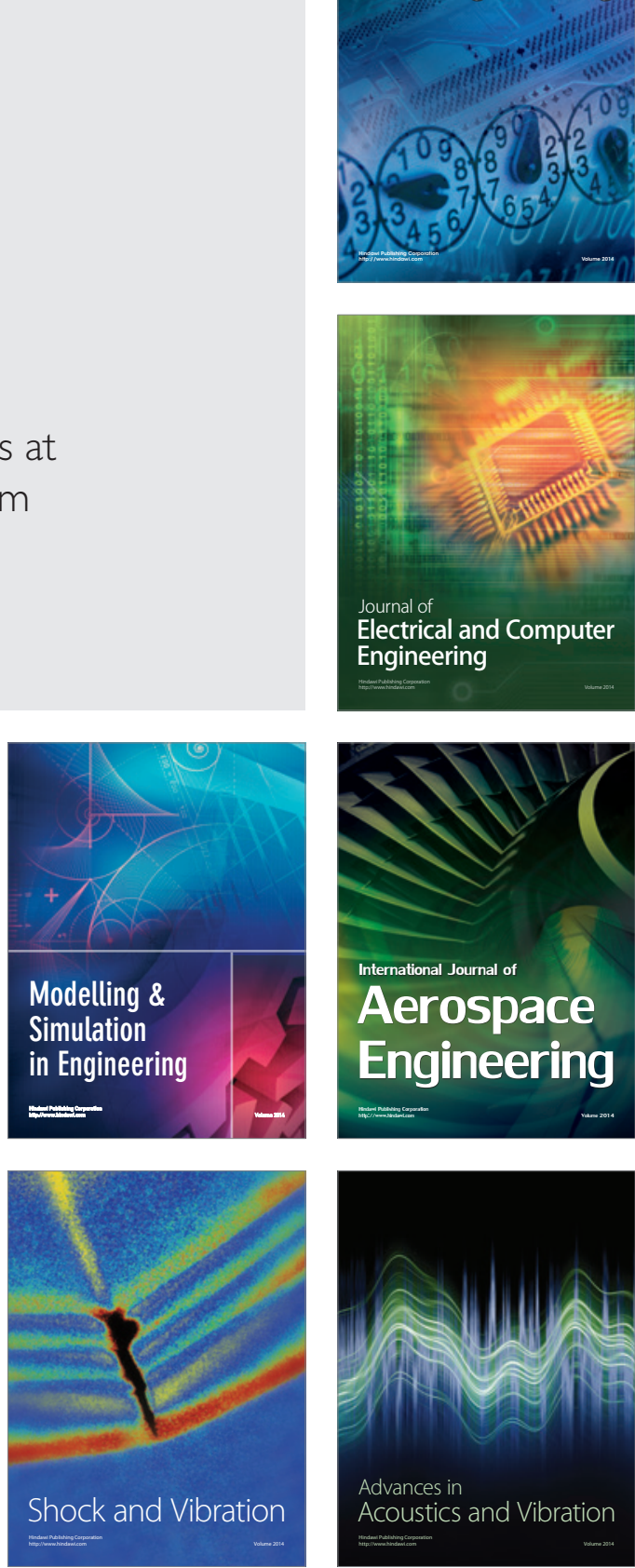